\section{artelogie}

\section{Artelogie}

Recherche sur les arts, le patrimoine et la littérature de l'Amérique latine

$12 \mid 2018$

Idiosyncrasie de l'indigénisme en Amérique latine.

Pluralité des sources et des appropriations extra-

latino-américaines

\title{
L'art indigéniste au féminin : Julia Codesido, Elena Izcue et Carmen Saco
}

Isabelle Tauzin-Castellanos

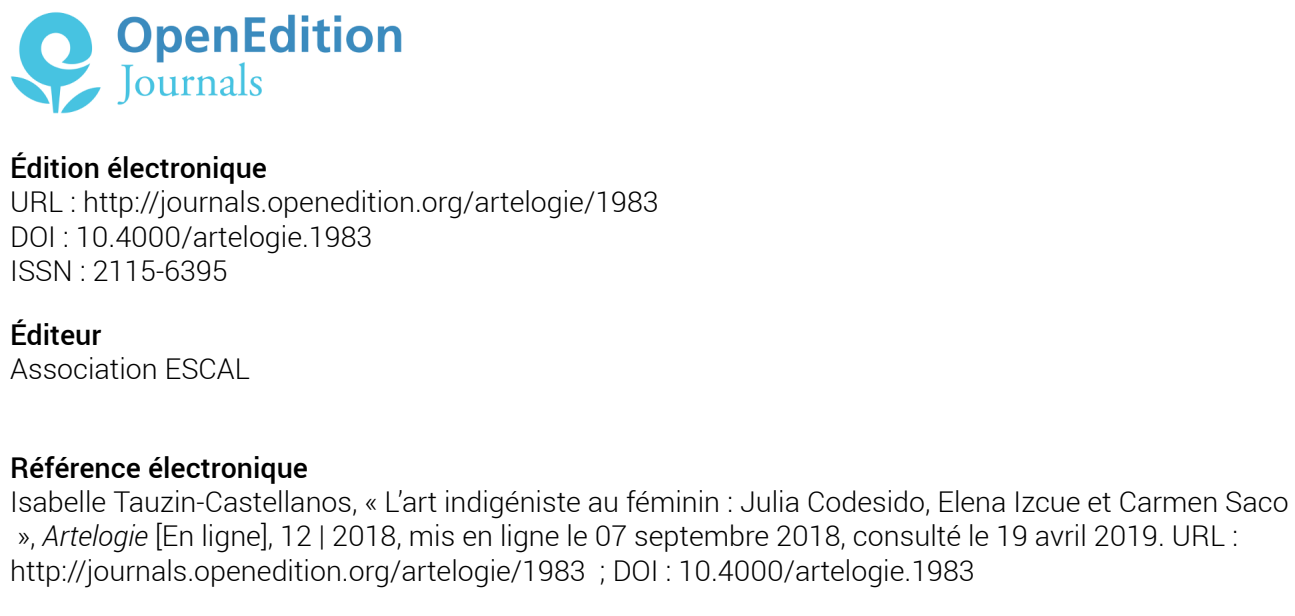

Ce document a été généré automatiquement le 19 avril 2019.

Association ESCAL 


\title{
L'art indigéniste au féminin : Julia Codesido, Elena Izcue et Carmen
}

\section{Saco}

\author{
Isabelle Tauzin-Castellanos
}

1 Pour le grand public, l'art latino-américain des années 1920-30 est assimilé au muralisme mexicain. Le projet indo-américain impulsé par Joaquín Torres García (1874-1949) concerne essentiellement le Rio de la Plata ${ }^{1}$, Buenos Aires étant alors la plate-forme de la vie artistique sud-américaine. Émigrée en Argentine, Gertrudis Chale (1898-1954) s'est enfoncée dans les territoires andins et a rencontré en 1945 à Lima les promoteurs de l'indigénisme péruvien José Sabogal (1888-1956) et Julia Codesido (1883-1979), à l'occasion de ce voyage à la recherche d'une «autochtonie non européenne dont elle mesur[e] la distance à l'aune de sa propre européanité» (Poloni-Simard, 2014 :9); la vision de Chale ressort assombrie de son pèlerinage: « J'ai vu l'Indien [...] la désolation de la puna dans les yeux et dans l'âme la tristesse énorme d'une race brisée dans son destin humain» (ibid.). L'indigénisme andin tardivement représenté en Équateur par Osvaldo Guayasamin (1919-1999) et en Bolivie par Marina Nuñez del Prado (1910-1995) a d'abord été encouragé au Pérou comme exaltation identitaire et esthétique d'une résistance pluriséculaire; l'implantation à Lima a sans doute nui à la visibilité du rayonnement dans l'ensemble du pays (Cajamarca, Cusco et Puno). Dans un article récent, Michele Greet étudie la présence latino-américaine dans les salons parisiens de l'entre-deux guerres (Salons de Paris, d'Automne, des Tuileries, des Surindépendants, des Vrais Indépendants...) ; elle relève la présence d'un seul artiste péruvien Felipe Cossío del Pomar ${ }^{2}$ dans les registres de la Société des Artistes Français, pour le portrait d'un couple d'Indiens («Descendants d'incas »).

2 Or, Paris a attiré les plus grands peintres de ce pays : le réaliste Ignacio Merino a étudié auprès de Monvoisin et Delacroix et a vécu jusqu'à sa mort à Paris en 1876; Daniel Hernández, premier directeur de l'Ecole des Beaux-Arts du Pérou (ENBA) a eu une carrière glorieuse à la Belle Époque pour ses portraits impressionnistes de blanches jeunes femmes primés à l'occasion de l'Exposition Universelle de 1900 à Paris. 
3 Le coût du voyage transatlantique et la vie parisienne n'était pas envisageable pour tous les jeunes artistes. D'origine modeste, José Sabogal vécut au jour le jour entre l'Italie, la France, l'Espagne de 1908 à 1910; revenu comme matelot à Buenos Aires, c'est en Argentine qu'il peut se former techniquement aux Beaux-Arts puis il est recruté comme professeur de dessin à Jujuy. La contemplation des vestiges préhispaniques, la vision des constructions monumentales de Tiahuanaco et de Cusco jouent le rôle de révélateurs ainsi que la fréquentation d'un groupe d'intellectuels convaincus de la supériorité inca contre le discours dominant de Lima qui valorise tout ce qui n'est pas local. L'École de Cusco ainsi nommé par l'intellectuel Francisco García Calderón s'est forgé à la suite de la grève des étudiants contre la perpétuation d'enseignants et d'enseignements scholastiques en 1909. Le renouvellement méthodologique fut impulsé par Alberto Giesecke, philosophe et économiste venu des Etats-Unis, chargé de rouvrir l'université par le président Leguía, et qui «introduisit des méthodes scientifiques d'observation, d'analyse et de recherche de données [...] Pour la première fois au Pérou la population indienne devenait objet de réflexion et d'étude scientifique [...] les études historiques et archéologiques réalisées par les étudiants leur firent prendre conscience de la valeur $\mathrm{du}$ passé de leur ville » (Dancourt, 1997 : 128-129).

4 C'est dans cette ville en pleine effervescence intellectuelle qu'arriva Sabogal en provenance de Jujuy et qu'il fut inspiré par la revendication identitaire. En 1919 après six mois passés à Cusco, Sabogal s'installe à Lima et déclare à l'occasion de sa première exposition: «Mes portraits sont avant tout caractère, esprit, race : ils représentent plus qu'une misérable individualité, toute une généalogie, un processus biologique complet, la documentation d'une époque de notre histoire. Je ne suis pas intéressé par le portrait individuel pour le seul mérite de l'exactitude du trait» (Tauzin-Castellanos, $2017: 433$ ).

5 La vie artistique est singulièrement dynamique comme dans tout le continent à l'orée des années 1920 et cette vitalité va s'amplifier avec l'émergence des indigénismes. Selon Gustavo Buntinx (2003:18) « les indigénismes (le pluriel s'impose) essaient de répondre à partir de la culture et des politiques de représentation originelle aux brusques renversements (physique, économique, psychologique...) éprouvés de manière convulsive par de vastes secteurs intermédiaires d'origine provinciale ». L'hétérogénéité définit en effet la peinture indigéniste tout comme la littérature indigéniste, dédiées à l'Autre, aux autres, les « indigènes» terme qui s'impose à la fin du xix siècle, alors que les artistes et auteurs indigénistes seront eux, créoles ou métis et en aucun cas indiens du fait de la distance économique et culturelle entre les deux mondes, urbain et paysan, hispanophone ou monolingue quechua ou aymara, de culture écrite ou orale. Par un effet spéculaire, après avoir reçu les leçons et admiré les modèles européens, les artistes indigénistes visent à renforcer le sentiment identitaire grâce à la contemplation de la différence et à l'affirmation de l'enracinement dans le territoire national par le biais de l'œuvre d'art.

6 L'indigénisme est un mouvement multiple qui concerne au seuil des années 30 toute la production artistique et culturelle (architecture, arts appliqués, peinture, photographie, musique, théâtre, littérature...). Il est l'expression à la fois, d'un discours officiel et d'un discours radical qui revendiquent de manière parallèle l'autochtonie. Pour les intellectuels de l'époque, il s'agit de valoriser « la Race » (le terme « race » n'est pas banni comme il le sera après le désastre du nazisme). L'indigénisme correspond alors à l'exaltation de l'héritage culturel indépendamment des influences européennes; c'est une 
forme de révolution culturelle pacifique contre la perduration de l'admiration et du pastiche du Vieux Monde.

7 Sur le modèle des muralistes mexicains, confrontés au bouleversement idéologique des codes culturels consécutifs à la Révolution de 1910, l'artiste se doit d'assumer et d'exalter la différence, comme signes identitaires du Pérou; l'indigénisme est andin et de manière extensive, bolivien et péruvien (le monde amazonien est le grand absent jusqu'aux années 50). José Sabogal est le chantre de cette fierté nationale qui triomphe avec lui, lorsqu'il accède à la direction de l'ENBA (1932). José Carlos Mariátegui résume précocement l'œuvre du fondateur de l'indigénisme péruvien :

8 «... dans le mouvement spirituel d'un peuple, les images sont parfois l'expression culminante. Les images génèrent des concepts, de même que les concepts inspirent des images. Sabogal apparaît ainsi, par son travail, étranger dans son intention à toute transcendance idéologique, comme l'un des constructeurs de l'avenir de ce peuple » ( Mundial, 1928, 28 juin, « La obra de José Sabogal »).

9 La production de José Sabogal a fait l'objet d'une rétrospective exceptionnelle en 2013 au Musée d'Art de Lima, impulsée par Natalia Majluf, alors directrice du MALI.

Dans l'environnement de Sabogal et dans la mouvance indigéniste émergente, pour contribuer à sortir de l'ombre les oubliées de l'histoire de l'art, dans un premier temps, j'évoquerai les artistes plasticiennes reconnues au début des années 20 : Elena Izcue (1889-1970) et Carmen Saco (1882-1948).

11 Dans un deuxième temps, va s'imposer l'œuvre de Julia Codesido. C'est dans le sillage de la revue Amauta qu'a lieu en 1929 la première exposition de Codesido. La libération de la peinture réaliste et le cheminement vers l'abstractionnisme explique le rayonnement de l'œuvre par-delà l'éclipse de l'indigénisme qui se produit dans les années 40 avec la contestation des Indépendants dont Ricardo Grau (1907-1970), influencé par Lhote et Léger et revenu au Pérou en 1937. Codesido a exposé au Palais des Beaux-Arts de Mexico en 1935, puis dans de nombreuses galeries du continent américain, à Paris en 1939 et en 1953, et à Barcelone en 1965. Son œuvre a fait l'objet de plusieurs ouvrages parus au Pérou (1986, 1990, 2010), dans l'attente de la réouverture de sa maison transformée en musée dans le district de Pueblo Libre ainsi que de la numérisation des fonds conservés par la Fondation Codesido. À quatre-vingt-treize ans, en 1976, Codesido reçut la tardive consécration de l'État péruvien, le Prix National de la Culture. Les sources iconographiques, qui ne sont pas toutes accessibles, du fait de la faiblesse des moyens affectés aux musées péruviens et de l'importance des collections privées, exposent des représentations sociales contemporaines révélatrices à la fois de la sensibilité de l'artiste et indissociables de l'environnement.

\section{Elena Izcue et Carmen Saco : des carrières tronquées}

Julia Codesido n'est pas la première peintre de l'histoire du Pérou. Sa professionnalisation lui donne une visibilité dont reste privée pour le siècle précédent Rebeca Oquendo de Subercaseaux ${ }^{3}$. En 1925, deux gros volumes sont publiés à Lima dédiés à «la femme péruvienne à travers les siècles». Dans une perspective féministe, mais avec les limitations d'une commande officielle, l'auteure, Elvira García y García propose les noms de vingt artistes plasticiennes, après avoir rencontré certaines de ces jeunes femmes et 
visité l'Ecole Nationale des Beaux-Arts de Lima en compagnie du directeur, Daniel Hernandez, peintre officiel du régime leguiiste ${ }^{4}$.

La biographie la plus développée est consacrée à Elena Izcue (1889-1879), qui constitue avec sa sœur Victoria un cas singulier dans un univers où le prestige familial force la carrière, et où la formation artistique correspond à un temps d'attente avant l'entrée dans la vie conjugale, au sein de la bonne société. Elena Izcue, dépourvue du réseau aristocratique indispensable pour être reconnue, enseigne le dessin à de jeunes enfants et est élève de l'ENBA ouverte en 1919.

En 1921, dans le contexte de la célébration du bicentenaire de l'Indépendance du Pérou, Elena Izcue reçoit une commande officielle pour le Musée National où elle scénographie une salle néo-inca. Elle bénéficie dès lors de soutiens public et privé pour se former et créer. Elle travaille aux côtés d'un magnat péruvien de la canne à sucre, collectionneur de pièces archéologiques exhumées sur le littoral, Víctor Larco Herrera, proche du pouvoir politique; Leguía est un homme de la Côte tout comme Larco, les États-Unis sont le modèle économique à suivre et en capacité d'investir dans cette Amérique d'en bas, qui n'est pas malmenée par des révolutionnaires. Il s'agit de proposer «une matrice culturelle générale et cohérente qui abolisse l'hétérogénéité des styles du Pérou précolombien et affirme en même temps l'existence d'un 'art péruvien', lequel fonctionnerait comme un héritage spirituel générant un fort sentiment d'historicité5 " (Vargas Pacheco, 2011 : 163).

En fait d'art décoratif péruvien et d'art inca, les motifs stylisés par Izcue (oiseau, poisson, renard...) sont inspirés des cultures côtières, figuratives, tandis que les restes archéologiques incas se distinguent par les éléments géométriques, la combinaison de lignes et de formes abstraites, également retrouvés sur les keros à vocation cérémonielle. Dans «Una visión del Perú a través del arte decorativo » Vargas Pacheco rappelle en outre la préface de l'intellectuel péruvien Ventura García Calderón à l'édition parisienne signée par Elena Izcue de El arte peruano:

"l'Europe a été notre obsession, nous méprisions un peu comme des conquérants ces restes de [notre] antiquité [...] à force de recevoir de beaux ouvrages documentaires imprimés aux États-Unis, à Paris ou à Berlin, l'art des Incas nous est apparu comme une chose vivante, dont la tradition est seulement interrompue » (Vargas Pacheco, 2011 : 153).

Vargas Pacheco suggère donc que «les nouveaux intérêts européens pourraient aussi avoir joué un rôle dans la revalorisation locale de ce qui est spécifique ('lo propio') » (ibid .). La curiosité pour le «primitif» ou «art premier» du point de vue européen est ambigüe. Elle obéit et est à la fois une réaction à la culture coloniale renforcée par le néoimpérialisme dominant. L'usage industriel et commercial, éloigné du contenu religieux associé aux représentations préhispaniques, pose problème. Si ces critiques concernent plus globalement la nature de l'art décoratif, l'oubli dont les sœurs Izcue ont été victimes, s'expliquent aussi par l'éloignement puisqu'elles firent carrière en Europe et aux ÉtatsUnis, et parce qu'elles représentaient la «Patria Nueva», le renouveau impulsé par le gouvernement Leguía autoritaire et rétrograde au fur et à mesure des onze années d'une présidence ploutocratique.

Elvira García y García présente aussi Carmen Saco (García y García, 1925 : 571-573) : « un cas unique parmi les artistes du Pérou ", issue d'une famille de notables, élève de l'ENBA et «sculptrice géniale». Carmen Saco (1881-1948) bénéficie d'une bourse du gouvernement Leguía pour se rendre en Europe en 1925. García y García, au lieu des 
circonvolutions fréquentes dans son histoire des Péruviennes, explicite l'engagement politique de Saco:

19 "Quant aux questions idéologiques, Carmen Saco sort tout à fait du commun. Socialiste très avancée, elle peut être considérée comme révolutionnaire, pour arriver à ce que règnent l'ordre et la justice. Elle-même disait:- Mon art, comme art, je ne peux pas le juger, mais ma volonté, mon désir essentiel est de faire de l'art prolétaire. Je veux aller vers le peuple, parce que je trouve en lui la source de mon inspiration, parce que je l'aime et parce que je ressens à travers lui ». (Ibid.)

20 Saco prévoit de fonder « sa propre école, inspirée de tout ce qu'elle a vu et admiré ». Saco a effectué plusieurs voyages et est allée au-delà de «l'Europe classique, occidentale et bourgeoise, but de tous les voyages de plaisir et d'études des rastaquouères de l'Amérique» (Amauta, $\left.\mathrm{n}^{\circ} 15,1928\right)$. Le chroniqueur regrette que Saco se contente d'esquisses, du fait de ses déplacements incessants. Deux céramiques («Maquette de paysans » et "Buste de métisse ») illustrent la note biographique. Saco se rend en Union Soviétique d'où elle écrit un reportage passionné par la vitalité du peuple qu'elle découvre (Amauta, «La ciudad mística ») et l'adoration posthume unanimiste à l'égard de Lénine. Saco a aussi séjourné en France; la mort de Bourdelle en 1929 est l'occasion de comparer le sculpteur disparu à Rodin:

21 "L'œuvre de Bourdelle exprima une sensibilité attardée, rajeunie par une passion intellectuelle. Elle est dépourvue d'une couleur définitive et pérenne, parce que son œuvre est celle d'un primitif dépourvu d'émotion. Et parce qu'il a essayé de nous rappeler le temps solennel des mythes et des symboles, à l'époque du vertige des avions, de la vie effrénée des automobiles, des convulsions sociales dans lesquelles les esprits empreints d'atavisme se récréent dans l'instant et suivent des voies distinctes ». (Amauta, 1929, 26 : 56)

22 Curieusement Bourdelle est défini comme primitif; le terme est péjoratif à son égard, comme l'anti-modèle pour la sculptrice péruvienne. En Espagne, à l'École pratique de céramique de Manises, Saco séjourne en même temps que le céramiste paraguayen Julián de Herrería d'abord inspiré par les cultures préhispaniques avant de se consacrer à la représentation des types populaires paraguayens. Une statuette «India en reflejo de oro» témoigne de cette étape de formation dans Amauta ( ${ }^{\circ} 23$, mai 1929, p. 20).

\section{L'œuvre de Julia Codesido dans Amauta}

Carmen Saco rend compte dans Amauta ${ }^{6}$, la revue dirigée par Mariategui, l'idéologue socialiste promoteur de l'avant-garde péruvienne, de la première exposition individuelle de Julia Codesido. Alors que Saco sera happée par le militantisme en particulier pour le Secours rouge, Codesido ne cessera d'évoluer dans ses pratiques artistiques.

Carmen Saco publie en décembre 1929 « Suggestions de l'art de Julia Codesido » (Amauta, $\mathrm{n}^{\circ} 27$, novembre-décembre 1929, p.17-20). La présentation est illustrée par quatre tableaux choisis parmi les vingt-trois toiles exposées tandis que douze seront brièvement décrites à la fin du même numéro par le poète surréaliste Xavier Abril' .

Les quatre portraits féminins ont été ré-agencés par Saco dans son article, d'une manière différente de l'ordre de l'exposition qui eut pour cadre le nouvel espace mis à disposition par le gouvernement de Leguia, le Conservatoire national (Academia Nacional de Música). «Cecilia », « Argile dorée » (Arcilla dorada), « Métisse de Cusco » (Chola Cuzqueña), « Petite 
Chinoise " (Chinita) sont les portraits reproduits dans ce numéro d'Amauta. Le choix de Saco, son interprétation de l'œuvre de Codesido est immédiatement idéologisé. Elle introduit ainsi les portraits: "[Codesido] embrasse dans son ensemble des races antagoniques, complexes, distantes, parmi lesquelles les accords ont toujours été rompus, les rapprochements ont toujours été désespérés. Mais cette peintre schismatique a réuni les éloignements implacables, avec une énergie et une autorité nullement féminines » ( Amauta, 27, 1929, p. 17).

Saco décrit les toiles qui ont vocation à mettre en scène "notre tragédie raciale, notre drame biologique [d'où] germera notre nouvelle vie, notre nouvelle civilisation enrichie grâce aux dons spontanés et mutuels" (id., p. 18). Les mots "dons" et "mutuels" renvoient à la réciprocité andine, la solidarité collective et la vision idéalisée propre aux années 20 de l'empire inca, considéré comme un communisme agraire dignes d'admiration et d'imitation.

27 La couleur est la caractéristique essentielle que Saco observe dans la peinture de Codesido :

28 "Les tableaux de Julia Codesido nous apportent la couleur des races aborigènes dans une vague qui porte comme liquides, des stries blanches, noires et jaunes. Et la vague cuivrée, énigmatique est chez elle dans l'attente d'un nouvel accord, l'arrivée de sa progression dans la nouvelle évolution. Dans l'exposition de Julia Codesido, la vague sombre striée est à sa place, car c'est son milieu géographique depuis la naissance [...] L'exposition de Julia Codesido [...] donnera un nouveau sens social à la femme, reléguée jusqu'à aujourd'hui aux obscures tâches mécaniques de dépoussiérer les bibelots et de changer les fleurs fanées du salon ». (Ibid. pp. 19-20). Le premier portrait «Cecilia» $\left(\mathrm{n}^{\circ} 16\right)$, exposé tout à côté de celui de José Carlos Mariátegui également de Codesido, est commenté ainsi par Xavier Abril : " 'Ceciliaa'. Personnalité qui se confond avec son paysage. Dans cette toile, triomphe la technique la plus difficile du portrait contemporain. Mis à part l'attrait de la modèle et de la réalisation artistique, il serait bon de considérer cette œuvre comme un véritable succès du portrait esthétique ». (Amauta, $\mathrm{n}^{\circ} 29,1929$, p. 100).

31 Cecilia Urteaga est représentée de trois quarts, dans la partie gauche du tableau. Un effet de symétrie est créé avec le cactus élancé, dans la partie droite. Cecilia représente le Pérou de la Côté, l'austérité du désert côtier au milieu duquel est située l'oasis de Lima. La posture est hiératique, la plante comme seule compagnie transfigure l'identité de la jeune femme, de même que Mariategui le maître à penser (Amauta), est représenté entouré de livres. Codesido est l'auteur de la couverture des Sept essais d'interprétation de la réalité péruvienne, la Bible de la génération socialiste indigéniste, les deux termes fonctionnant alors comme synonymes'. La couverture est inspirée de l'architecture inca, le trapèze au centre et la forme de gradins suggérant les temples incas couleur terre. 


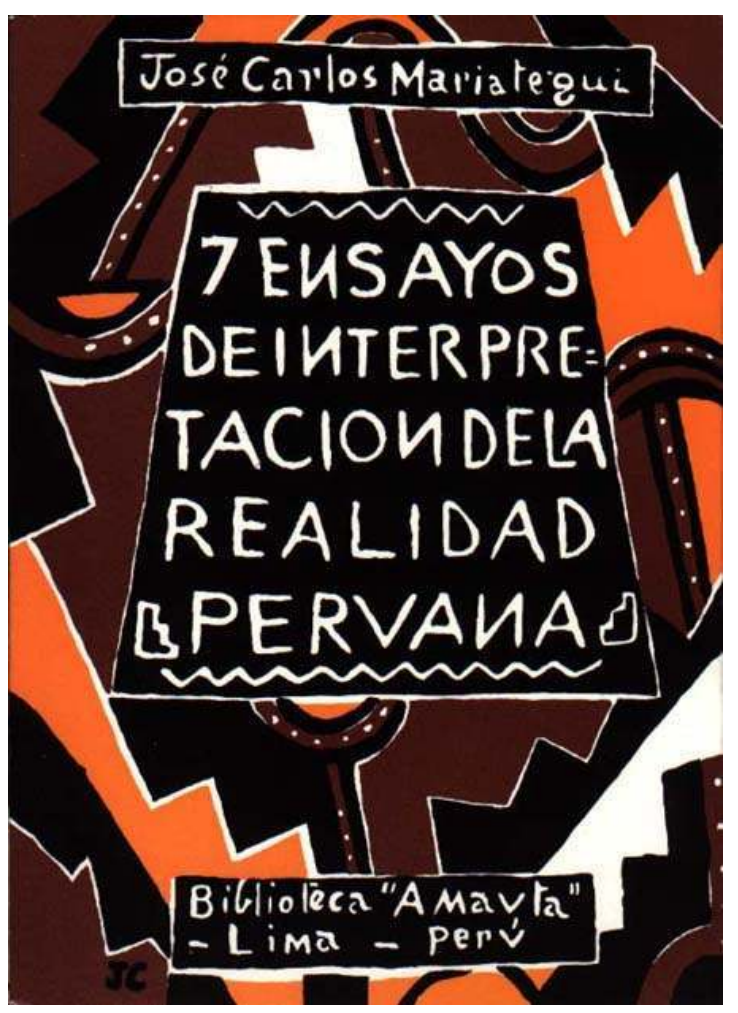

FIgURE 1: JULIA CODESIDO, 7 ENSAYOS DE INTERPRETACIÓN DE LA REALIDAD PERUANA 1928

Le deuxième tableau sélectionné par Carmen Saco, « Argile dorée » (« Arcilla dorada ») est une grande toile ultérieurement reproduite sous le titre plus explicite «Indienne accroupie » et qui va de pair avec «Argile quechua» (rebaptisée plus tard « $\mathrm{Nu}$ ») et "Métisse du Cusco », tous trois dans Amauta.

La même jeune femme a posé dans les trois tableaux, avec des atours différents, signifiant une identité locale à valeur exemplaire. La «métisse du Cusco»- «chola»- porte la coiffe circulaire de Chinchero, la « vallée sacrée des incas » inspiratrice de José Sabogal et son célèbre «Chef indien » (Varayoc,1925). 


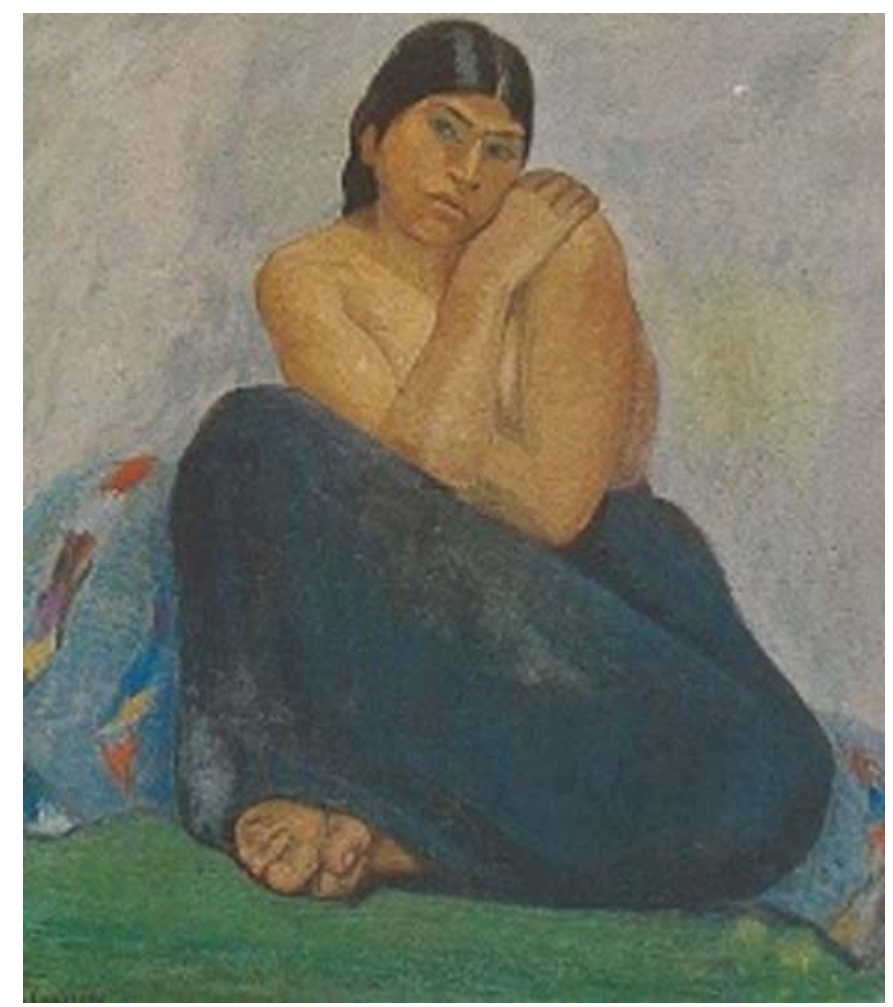

Figure 2. :Julia Codesido, Argile dorée - Arcilla dorada (huile sur toile, $90 \times 79 \mathrm{~cm}$ )

«Argile dorée » de Codesido (dont le titre insiste sur la matière et la couleur) est un portrait qui plante au centre son sujet et comble l'espace. La jeune femme à l'allure vigoureuse est toute en courbes, sur un fond gris bleu. La forme triangulaire du corps, transcendance de la puissance tellurique, se dresse et s'apparente au profil massif des Andes, animés par les esprits des montagnes, les apus. Le regard scrute le spectateur sans dévoiler de sentiment. Le corps féminin est à demi-nu, enveloppé dans un morceau de tissu teint en indigo. Les couleurs complémentaires du bleu violet et de l'ocre orangé mettent en évidence le buste musculeux. La puissance virile est déniée par la gestuelle, les bras croisés cachent et suggèrent les seins, tandis que les pieds mal dissimulés au premier plan disent le dénuement économique et la proximité de la terre dont la jeune femme est la créature. Le spectateur devine les tresses, qui a contrario seront en évidence dans l'autre portrait intitulé "Argile quechua" de même que les seins et les jambes découvertes de la jeune femme, dans une posture hiératique, double humain de la céramique préhispanique humanisée placée à côté et valorisée depuis le xixe siècle et au xxe siècle par les découvertes des archéologues Max Uhle et Julio C. Tello. La plasticité du corps féminin indien que déclare ce tableau de Codesido, est un discours contraire à l'académisme qui exaltait blancheur et blondeur des corps européens et américains, et est inspiré cependant de la tradition antique, une version féminine du scribe égyptien assis en tailleur, mais c'est aussi un corps dépouillé et sexué à l'inverse du potier indien dans un poncho noir du peintre romantique péruvien Francisco Laso (1855) 


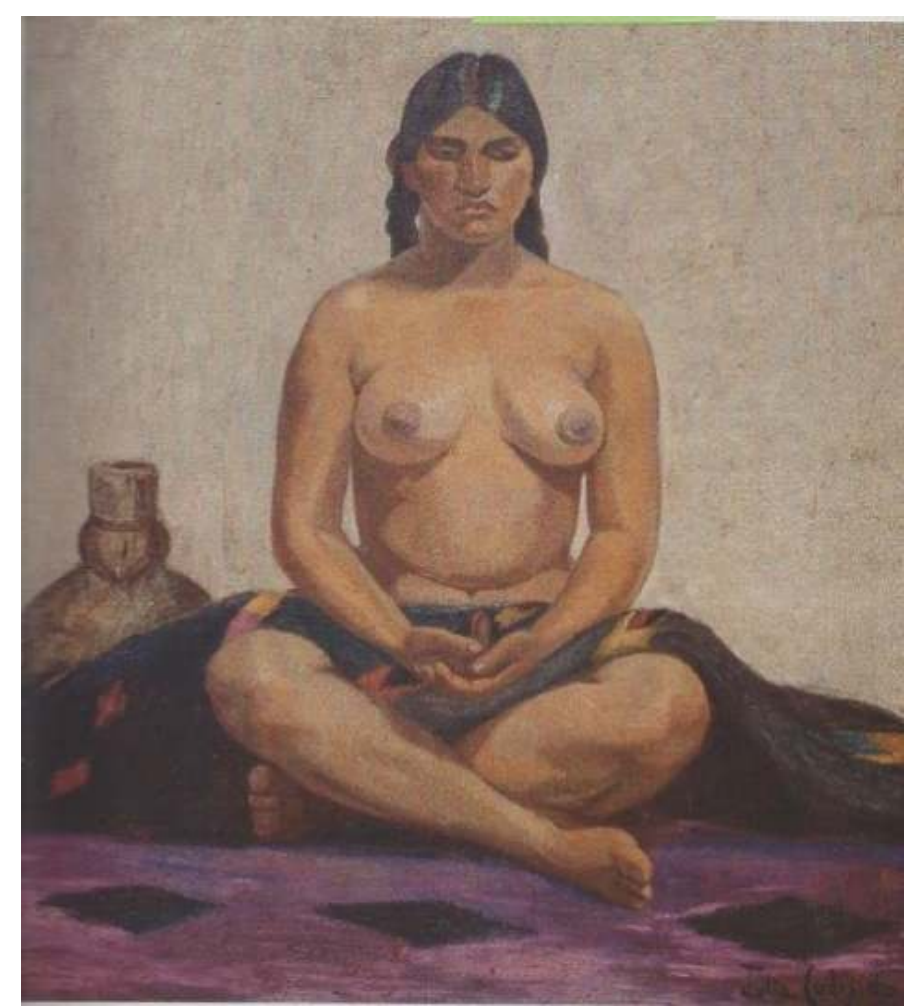

Figure 3 Argile quechua - Arcilla quechua - Desnudo (Huile sur toile 110X107cm)

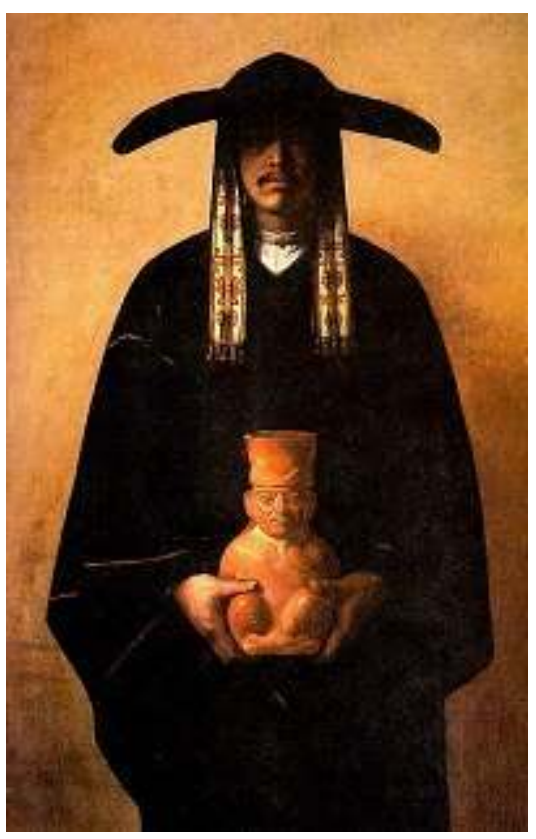

Figure 4: F. Laso (1855): Potier indien (Alfarero indio). Museo de Arte de Lima (Huile sur toile 88X138 $\mathrm{cm})$

La difficulté de poser nue a été rapportée par Carlota Carvallo ${ }^{10}$, alors élève de l'ENBA et qui accepta de répondre à la demande de José Sabogal. Carlota Carvallo se forme dans l'environnement de Sabogal ; outre les cours de l'École Nationale des Beaux-Arts où elle obtiendra le premier prix de peinture en 1933, elle évoque l'émulation à l'égard de Sabogal, l'amoureuse admiration de Codesido, et les heures passées à peindre des toiles de fond pour une exposition en 1931. La chevelure occultée dans « Argile quechua » est aussi 
signifiante, à l'opposé du portrait d'Alicia Bustamante ${ }^{11}$, coiffée à la garçonne et cigarette à la main (1924). La jouissance esthétique dans la représentation du corps féminin sera une constante des tableaux de Codesido, de ses tout premiers nus formellement insoumis, aux toiles allégoriques ultérieures comme « La terre » et « L'eau ».

Dans «Argile dorée » la céramique ancienne est remplacée par une mante de couleur bleue surlignée par une bordure arc-en-ciel qui renvoie à la cosmovision andine. Xavier Abril commentait : «Argile Dorée. Pudeur presque primitive à l'image du sens indigène du nu. Pudeur sans relation avec l'absence de vêtement mais chargée d'un fort érotisme du corps. Pudeur non cultivée, sans esprit décadent, sans miroir : pureté ». La nudité indienne est nature et innocence dans cette lecture, tandis qu'un autre nu sera "sensibilité et souffrance, désespoir presque de ne se sentir qu'un corps confronté à la vaine espérance romantique [...] légère nuance sensuelle qui naît dans les yeux comme un nuage cafard [sic]». (Amauta, n 29, 1929, p. 100). «Argile dorée » sera rebaptisée «Indienne accroupie » (« India en cuclillas », figure 2), l'allégorie initiale remplacée par le premier degré avec la dénonciation de la servitude indienne connotée par les mots du titre placés comme équivalents ( indien »= «accroupi »). La saturation des couleurs l'emportera plus tard dans l'œuvre de Codesido et la corpulence salutaire sera remplacée par des formes longilignes.

Deux ans avant l'exposition de décembre 1929, la revue Amauta avait déjà reproduit en noir et blanc six tableaux de Codesido et applaudi une œuvre qui «ne fleuretait avec aucune mode et dont par un mouvement naturel de l'esprit, les sujets sont presque tous autochtones "; la peintre contribue à "l'effort de créer un Pérou nouveau ", sans « jamais rester dans la note de folklore » (Amauta, $\mathrm{n}^{\circ} 11$, janvier 1928, pp. 9-10). Les tableaux dans Amauta ne sont pas représentatifs de la totalité de la production de Codesido, dans les années 20. Sous l'égide de José Carlos Mariátegui, le choix a été fait de montrer les toiles en harmonie avec l'orientation de la revue; Codesido est définie par "quelque chose d'ascétique ", comme "une mystique de son art » (ibid.), ce qui peut être interprété négativement comme l'a fait Eve-Marie Fell commentant « une pose statique, le regard fixe vers le lointain et dénué d'expressivité [...] une effigie, intemporelle et générique» (Fell, 2007: 610) ou a contrario valorisé par Nicole Fourtané qui décrit le dessin de Codesido pour le numéro 18 d'Amauta: " la rencontre de deux paysannes, avec leurs tresses, leurs chapeaux, leurs llicllas et leurs polleras dans un paysage agraire dominé de cactus. Les personnages se détachent en noir sur fond jaune, la couleur du soleil. La simplicité de cette composition est un hymne à la vie quotidienne de la Sierra " (Fourtané, 2017 : sp.). 


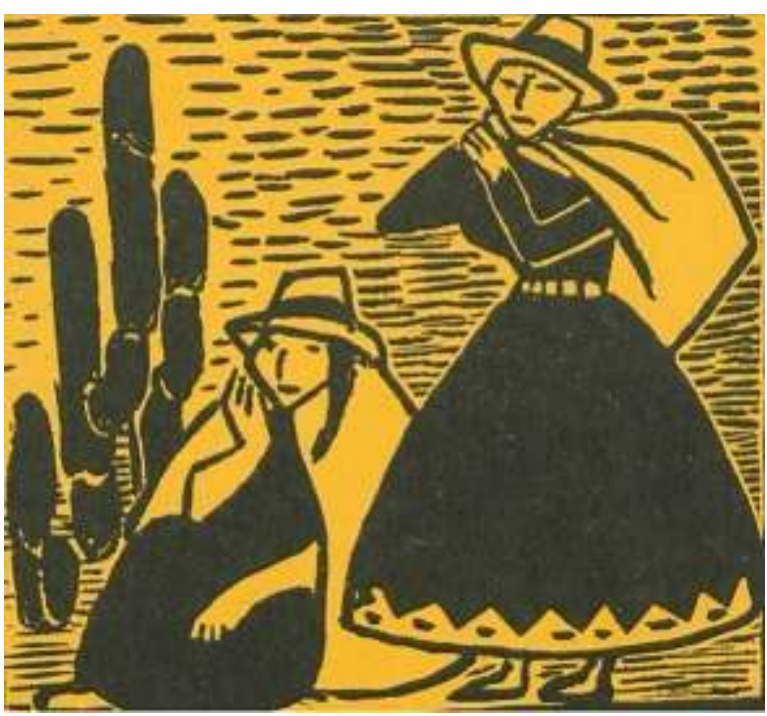

Figure 4: Couverture d'Amauta 18 - octubre 1928 - Julia Codesido

Le caractère naïf est volontaire et inspiré de la pyrogravure des calebasses tout aussi ocre, exaltation de l'artisanat traditionnel andin revalorisé par Sabogal et ses disciples au sein de l'Institut de l'Art Péruvien fondé en 1931.

Codesido contribuera grandement au projet de représenter l'art populaire, par des aquarelles, des temperas et des dessins «dans un style synthétique et sobre éloigné de simples reproductions [...] sa manière d'affronter la peinture contredisait le présupposé de copier un objet comme par simple calque ", cela afin de construire un « récit métis", observe Fernando Villegas qui inventorie 178 travaux de Codesido sur un total de 283 œuvres réalisées pour l'Institut de l'Art Péruvien (IAP) représentant des céramiques, des jouets, des statuettes, des retables et des keros (Villegas, $2006: 28$ ).

La première difficulté rencontrée pour reconstruire le parcours de Julia Codesido se trouve dans les informations contradictoires de certaines biographies malgré le fait qu'Eduardo Moll a levé tous les doutes sur sa date de naissance, le 5 août $1883^{12}$. Le nom de famille a subi des variations : "Codecido » est d'origine vénézuélienne; les premiers tableaux de Julia en revanche sont signés Codesido, dès le début des années 20. Le changement graphique qui marque l'identité visuelle par l'inscription $\mathrm{du}$ « $\mathrm{s}$ » au milieu du patronyme, a un sens en Europe du fait de la différence phonétique; au retour d'un séjour de dix-sept années (1900-1918), la peintre choisit son nom, mais la variante n'est pas reprise par les premiers critiques dans la revue Amauta qui publie en 1928 sept photos sous le titre "Art péruvien: Julia Codecido» («Tisseuse indienne», «Argile quechua », «La quena », «Indien aymara », « Bébé indien », « Portrait du romancier Augusto Aguirre Morales », « Métis de Cusco $\left.^{13} »\right)$.

Bernardino Codecido était arrivé avec sa famille en Europe, comme consul du Pérou à Lausanne en 1901, puis en poste à Liverpool en 1908, à Bordeaux en 1913 (20 janvier), au Havre à la fin de la même année 1913. C'est une époque de prospérité économique pour le Pérou en pleine modernisation tandis que la haute bourgeoisie latino-américaine va et vient entre Paris, Biarritz et Madrid. Julia Codesido résume son expérience artistique de jeunesse en très peu de mots, limitée à la visite du Prado et à la contemplation des tableaux du Greco, de Goya et de l'École Flamande ${ }^{14}$. La formation par la visite de musées ne paraît cependant pas suffisante pour comprendre la maitrise du geste dès les premiers portraits conservés du début des années 20. 

intègre dès son ouverture l'Ecole Nationale des Beaux-Arts impulsée par le président José Pardo et confiée à Daniel Hernánde $z^{15}$. De cette étape de formation académique, les traces sont variées. Le thème indien est très prégnant mais pas exclusif, comme on pourrait l'imaginer de par les reproductions dans la revue de José Carlos Mariátegui. Tout comme Sabogal, Codesido rapporte «un éblouissement» (Falcón, 1986: 18) de la première découverte des Andes. La représentation de types ethniques est rationnalisée a posteriori : «L'Indien péruvien est [...] une révélation humaine de force, de résignation, de patience et de foi. Son intelligence ancestrale est débordante » (id.). Le propos de Codesido est en même temps significatif de l'écart sociétal qui sépare l'artiste cosmopolite et son sujet: «Un grand homme de science de notre monde intellectuel qui l'a étudié à fond dit que ses vertus s'élèvent à un niveau respectable. Aujourd'hui encore [l'Indien] nous donne des enseignements surprenants aussi bien dans le domaine pratique que scientifique et spirituel » (ibid.). Alors qu'en Europe, l'insolite, le primitif et l'inconscient sont les mots clés du renouveau esthétique surréaliste, le Pérou présidé par Augusto B. Leguía a pour mot d'ordre la «Patrie Nouvelle», construire un nouveau pays et de nouvelles représentations du Pérou à l'occasion du centenaire de l'Indépendance (1921). C'est dans ce cadre que Codesido travaille essentiellement des portraits de ses connaissances aujourd'hui anonymes, à l'instar de Sabogal, tandis que les artistes originaires de Cajamarca Mario Urteaga et Camilo Blas se sont spécialisés eux dans les scènes de genre ${ }^{16}$. Codesido a également représenté des paysages urbains, espaces quasiment vides, hantées par des silhouettes avachies. Devenue professeur de l'ENBA, elle a pu voyager en toute autonomie, à partir des années 30, en Amérique grâce au soutien des Mexicains Orozco et Siqueiros, puis en Europe plus difficilement.

Le Petit-Palais conserve un dossier consacré à l'exposition que Codesido fit en 1953 à Paris. Ces archives qu'il nous a été donné de consulter, permettent de mieux appréhender les obstacles auxquels elle a été confrontée tout au long de sa carrière et que le regard rétrospectif a tendance à atténuer.

\section{Aller - retour vers l'Amérique}

Partie en 1918, Codesido est revenue d'abord à Paris en mars 1939. Elle évoque cette étape en 1966 : «Je me débrouillai seule dans cette entreprise, j'ai eu des difficultés à trouver un endroit pour mener à bien ce projet » (Falcón, 1987 :13). Codesido expose dans la galerie Barreiro, grâce au soutien de René Huyghe, conservateur du Louvre et qui avait réalisé un voyage à Lima, visité l'ENBA et manifesté son intérêt pour la peinture indigéniste et sa disponibilité pour soutenir les artistes péruviens. L'exposition eut lieu juste au moment de la déroute de la république espagnole, en mars 1939. La galerie Barreiro exposa en même temps des tableaux du peintre expressionniste espagnol José Gutierrez Solana, dont «La visite de l'évêque » avait été reproduite dans Amauta en 1928 et décrite par Carmen Saco. Une toile de la série «Indiennes Huancas» fut achetée par la Mairie de Paris et est actuellement conservée au Centre Pompidou 


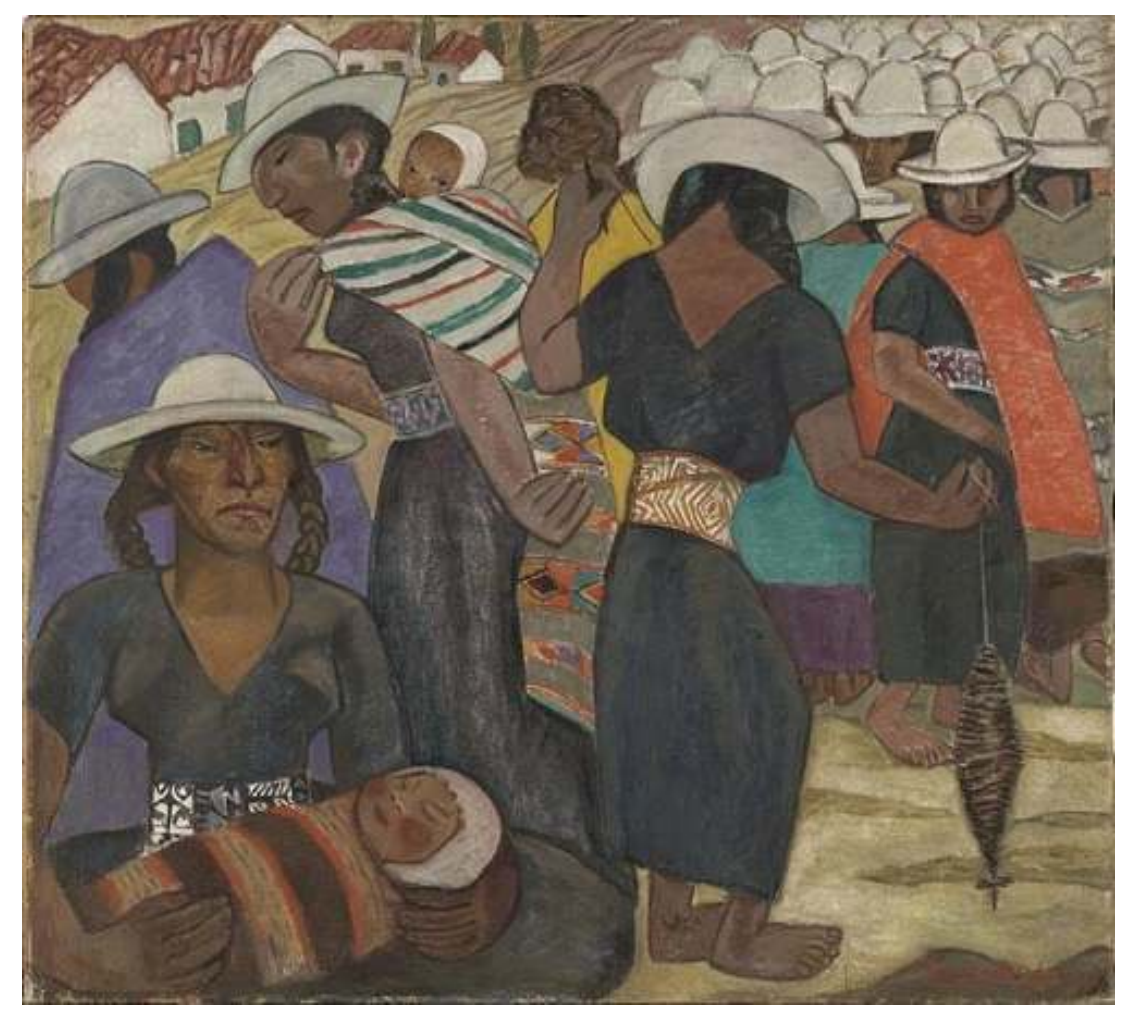

Figure 5: Indias huancas, 1931. (Huile sur toile, 106X116cm).

Centre Pompidou.

La maternité et le travail féminin, objectivés par le nourrisson et le fuseau, sont les sujets de cette composition où l'identité est forcément collective, marquée par l'habillement qui définit les Huancas. Le paysage est délimité dans l'angle supérieur, remplissant l'espace avec la série de coiffes blanches, à la façon des calebasses pyrogravées surchargées de détails. Le tracé est à la fois précis et faussement maladroit, l'impression de profondeur est favorisée par l'effet de contre-plongée que l'on retrouve dans les autres tableaux de 1931 (Fête de Huancahuari, Les bougies, Indienne Huanca) présentés lors de la deuxième exposition de Julia Codesido, à l'université nationale de San Marcos.

La seconde exposition à Paris eut lieu en 1953, sous le titre "Trois artistes sudaméricaines ». Les œuvres de Marina Nuñez del Prado et Irene Hamar furent exposées aux côtés de celles de Julia Codesido tandis que les Cubains Luis Martínez Pedro et Mario Carreño également prévus s'étaient désistés; tous les frais (transport, assurance, catalogue et affiche) étaient à la charge des exposants.

Ce sont trente-trois tableaux de petite taille que Codesido choisit cette fois de présenter; la modeste plaquette préparée à cette occasion, en noir et blanc, avec quelques vagues lignes d'André Chamson, alors directeur du Petit Palais, est la seule trace conservée ${ }^{17}$; elle nous a permis de constater l'ampleur de la rétrospective préparée par Codesido, les titres des toiles suggérant les trois visages du Pérou : la Côte, les Andes et l'Amazonie. 


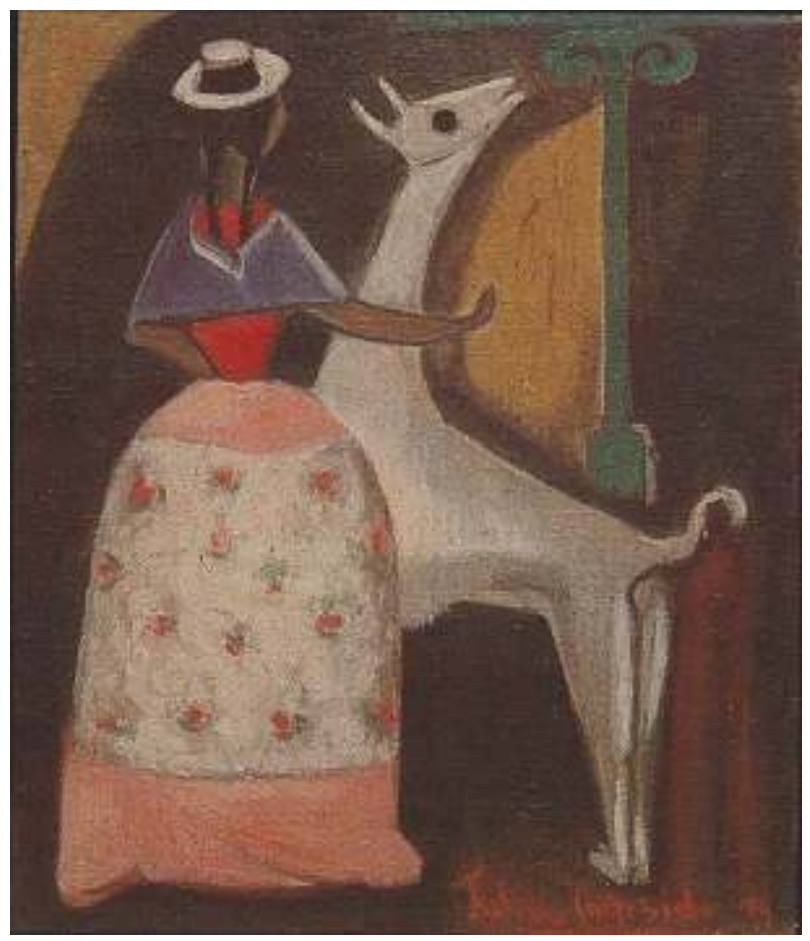

Figure 6: Indienne et lama (Una india con llama, 1949, huile sur toile, 48×36cm)

51 «Indienne et lama » a été peint en 1949 et reproduit en noir et blanc pour la plaquette de 1953. "Una india con llama ${ }^{18}$ » peut être considéré comme l'aboutissement de la quête indigéniste de Julia Codesido à l'orée des années 50, au moment où l'exode rural s'amplifie et où la question du métissage remplace la discussion sur l' «Indien ». Le trop-plein qui caractérisait les toiles indigénistes de Codesido est remplacé par une représentation allégorique du Pérou. Une jeune femme enveloppe de son bras un lama stylisé dont le regard capte l'attention. L'indienne est irréelle et archétypique à la fois : les nattes sous un chapeau blanc de céruse, le triangle du poncho indigo, le teint de bronze d'une part, mais aussi le corsage rouge carmin, la taille cintrée, la "pollera » rose surlignée par une sur-jupe blanche à fleurs donne au personnage le charme de l'extranéité, à la fois proche et lointain, avec un rendu naïf renforcé par la perspective curviligne. L'aquarelle « costumbriste » reproduisant avec la plus grande précision les habits traditionnels et les objets artisanaux pour préserver et transmettre les coutumes régionales dans le cadre de l'Institut d'Art Péruvien et du Musée de la Culture Nationale a été l'étape formelle préalable qui a aussi contribué à dépasser l'image misérabiliste pour une exaltation de la beauté qui passe par les couleurs de plus en plus vives et la géométrisation des corps, sur des fonds réduits à l'application d'une couleur. L'un des tout derniers tableaux de l'exposition de Paris a pour titre «Chefs indiens ", au moment où triomphe l'art brut de Dubuffet. Trop audacieux pour figurer sur la plaquette, la toile est aujourd'hui l'une des œuvres les plus connues de Codesido par la simplicité des formes, ponchos losanges rouges, chapeaux circulaires rouges de la communauté de Chincheros, comme variation $\mathrm{du} «$ Varayoc » de Sabogal (1925). 


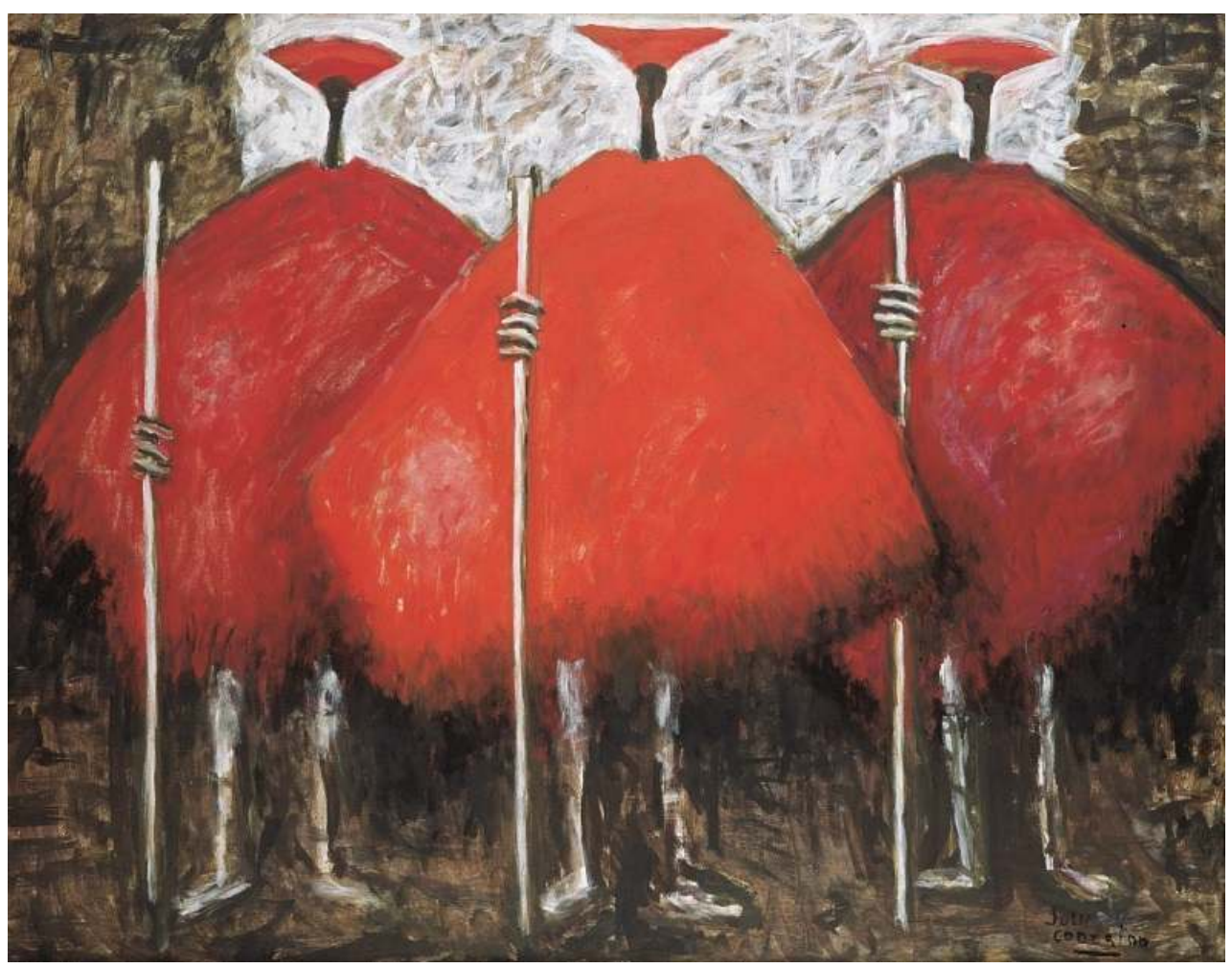

Figure 7. Julia Codesido: Trois chefs indiens (Tres jefes indios, huile sur toile, $121 \times 95 \mathrm{~cm}$. Pinacothèque Ignacio Merino, Lima)

Les bâtons rectilignes symbolisent l'autorité communale, sans plus de visage individuel; le reste du corps est minimaliste et renforce la verticalité du tableau. Les couleurs rouge et blanc suggèrent-elles la nationalité péruvienne? Un cercle lumineux à la touche morcelée derrière les trois têtes consacre les « varayocs ».

Codesido aboutit à une quête de l'élémentaire, à la traduction picturale de la spiritualité que lui apportent les voyages par-delà même les Andes, auprès des habitants de l'Amazonie qu'elle peint dans les activités quotidiennes que sont la pêche et le tissage et évoque avec ferveur à l'occasion de l'exposition de Barcelone (1966):

«Je suis fascinée par les gens de la forêt, je ne pourrais pas dire sauvages du fait de leur inclinaison au raffinement artistique, leurs habitudes pittoresques et originales en font des êtres aux qualités rares [...] Leur céramique et tissus imprimés sont comme leurs habits d'une finition technique curieuse et magnifique. Tout est fabuleux dans la forêt, la faune et la flore sont fantastiques ; [...] se plonger dans l'étude de la forêt est une chose sérieuse et fascinante». (Falcón, 1987 : 18-19) 


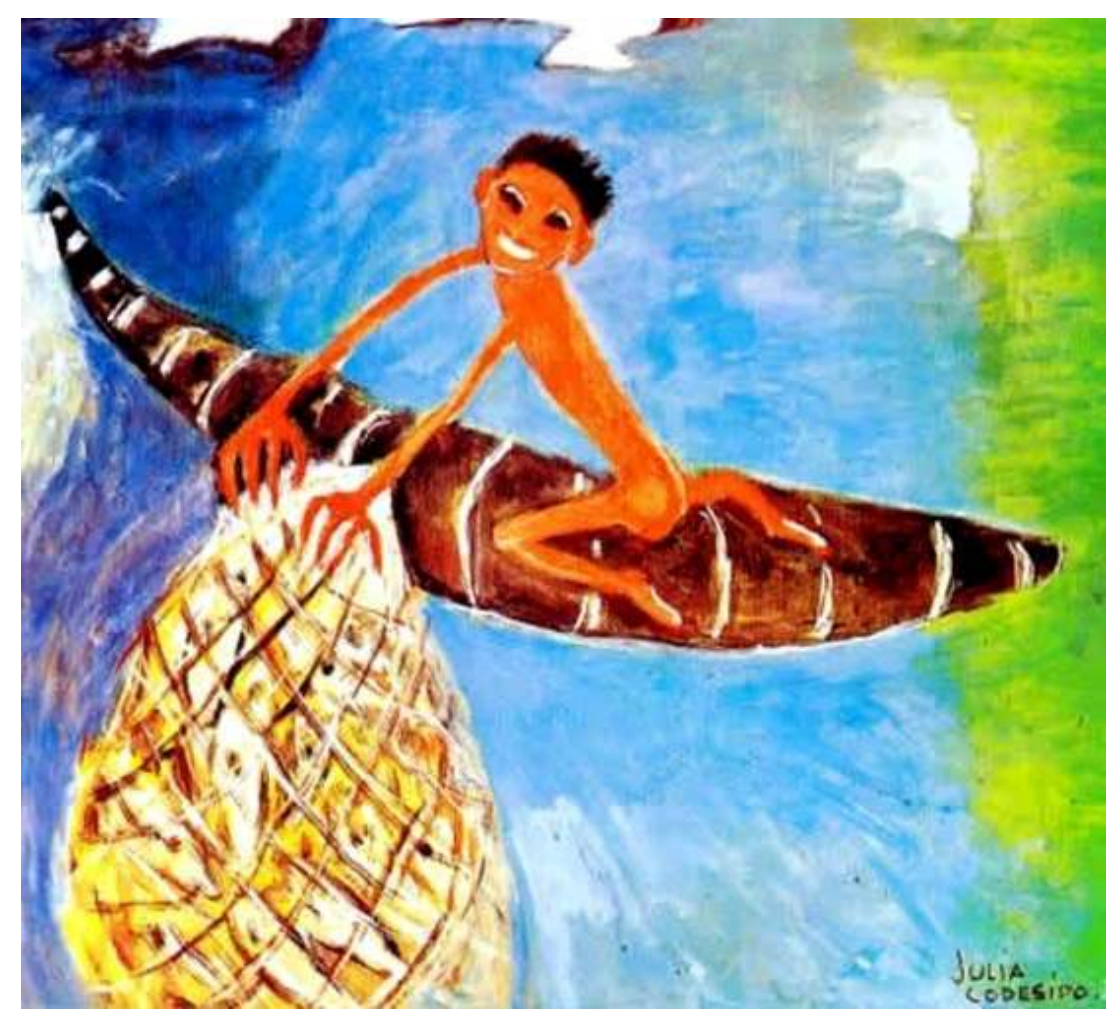

Figure 8. Julia Codesido, Le filet c. 1950 (La red. Huile sur toile, 64X64cm. Archive E. Moll)

Ce parcours trop rapide de l'œuvre de Julia Codesido est représentatif de la carrière de nombreux artistes plasticiens péruviens. La recherche de la reconnaissance, après l'étape de formation, a impliqué les voyages en Europe, les expositions internationales sur fonds propres dans des galeries souvent modestes. L'exploration de la correspondance de Codesido permettra d'apporter des éléments sur cette expérience, au-delà du fonds documentaire conservé par le Musée d'Art de Lima et consulté pour cette étude.

Une seule œuvre de Codesido est conservée dans le patrimoine artistique français. «Les indiennes huancas» illustre l'engagement artistique des années 30. La rencontre des muralistes mexicains d'une part, le travail quotidien comme professeure de l'Ecole Nationale des Beaux-Arts puis à l'Institut d'Art Péruvien fondé par Sabogal et qui conserva son autonomie après le départ forcé du père de l'indigénisme de l'ENBA, les voyages dans l'intérieur du Pérou de Codesido malgré l'enclavement du pays expliquent son évolution remarquable vers l'abstraction, qu'elle a toujours refusé tout en faisant sienne une phrase qu'elle répétait en français au nom de Mondrian: «C' est une porte ouverte à l'infini » (Falcón, 1986 :16). La peinture indigéniste ne s'est pas limitée à Lima, les villes des Andes - Cusco, Puno et Cajamarca - ont été des foyers d'autant plus actifs que l'obstacle de la distance est resté majeur jusqu'à la fin des années 40 , lorsque l'indigénisme commence à être contesté avant d'être définitivement déprécié au moment où la politique se tourne vers les communautés andines pour reconnaître leur existence. La reconnaissance de Julia Codesido arrive alors, puis la crise des années 80 replonge son œuvre dans l'oubli. 


\section{BIBLIOGRAPHIE}

Sources primaires

Abril Xavier, « Catálogo de la exposición de Julia Codesido », Amauta, 26, décembre 1929, p. 100.

Amauta, 1926-1930. $\mathrm{N}^{\circ} 1-30$. Consultation : Bibliothèque Université Bordeaux Montaigne.

Collection en ligne : http://digital.iai.spk-berlin.de/

Carvallo Carlota, Diario de 1931, inédit (communiqué par Sandro Patrucco)

García y García Elvira, La mujer peruana a través de los siglos, Lima, Imprenta americana, 1925.

Mariátegui José Carlos, «Julia Codecido, nota de la dirección con 7 fotograbados, Amauta, 11, janvier 1928, pp. 8-12.

Mariátegui José Carlos, «La obra de José Sabogal », Mundial, 28 juin 1928, en Obras completas, 1959, Lima, Amauta, Vol. 6, p. 92.

«Trois artistes sud-américaines 1953. Photos d'œuvres et Catalogue ». Musée du Petit Palais. Centre de ressources documentaires, Paris.

Consultation du dossier: Février 2018.

Saco Carmen, « Balance sumario de Bourdelle », Amauta, 26, septembre-octobre 1929, p. 56.

Saco Carmen, « Sugestiones del arte de Julia Codesido », Amauta, décembre 1929, 27, pp. 17-20

Saco Carmen, « Las exposiciones », Amauta, décembre 1929, 27, pp. 98-99.

Biographies et catalogues

Buntinx Gustavo et Luis Eduardo Wuffarden, Mario Urteaga: nuevas miradas, Lima, 2003, Museo de arte de Lima-Telefónica.

Falcón Jorge, Julia Codesido, Lima, 1987, Minerva.

Julia Codesido, Lima, 2010, El Comercio - Punto y Coma (ed).

Majluf Natalia et Luis Eduardo Wuffarden, Elena Izcue. Lima-Paris Années 30, Paris, Musée du Quai Branly, 2008.

Majluf Natalia et Luis Eduardo Wuffarden, Sabogal, Lima, MALI, 2013.

Moll Eduardo, Julia Codesido, Lima, 1990, Navarrete

Articles critiques

Carpio Kelly et María Eugenia Yllia, « Alicia y Celia Bustamante, la Peña Pancho Fierro y el Arte Popular », Illapa, $\mathrm{n}^{\circ}$ 3, 2006, pp. 45-60.

Dancourt Carlos, « Mouvements intellectuels fondateurs 'La escuela cuzqueña' ", América, 1997, 19, pp. 123-139. DOI: 103406/ameri.1997.1314

Fell Ève-Marie « Représentations iconographiques de la Nation dans Amauta. Pérou, 1926-1930, sous la direction de Jean-Louis Guereña, Images et transmissions des savoirs dans les mondes hispaniques et hispano-américains, Tours, 2007, Presses Universitaires François Rabelais, pp. 603-612. 
Fourtané Nicole « La réflexion de Manuel González Prada et José Carlos Mariátegui sur l'intégration de l'Indien à la Nation : Aspects idéologiques et esthétiques », Amerika 17, 2017.DOI : 10.4000/amerika.8372

Pachas Maceda Sofía, « Rebeca Oquendo. Un legado pictórico por descubrir », Illapa, n 7, 2010, pp. 11-20.

Poloni-Simard Jacques, « Le voyage des Andes des artistes du Rio de la Plata au XXe siècle », Artelogie, $\mathrm{n}^{\circ} 14,2014$, pp. 1-18. DOI : 10.4000/artelogie.1294

Tauzin-Castellanos Isabelle, « Representaciones del Inca Garcilaso de la Vega por José Sabogal», Revista de Crítica Literaria Latinoamericana, 2017, $\mathrm{n}^{\circ}$ 85, pp. 429-443.

Vaudry Élodie, « Usos políticos y culturales del recueil de adornos peruanos : el caso de Elena Izcue», M. Cardenas et I. Tauzin-Castellanos, Miradas recíprocas entre Perú y Francia, Lima, Universidad Ricardo Palma, 2015, pp. 329-345.

Vargas Pacheco Cristina, Una visión del Perú a través del arte decorativo, Mercurio peruano, 2011, $\mathrm{n}^{\circ}$ 524, pp. 151-173. https://dialnet.unirioja.es/ejemplar/283312

Villegas Fernando, «El Instituto de Arte Peruano (1931-1973): José Sabogal y el mestizaje en arte », Illapa, 3, 2006, pp. 21-34.

http://revistas.urp.edu.pe/index.php/Illapa/article/view/1150

\section{NOTES DE FIN}

1. Jacques Poloni-Simard, "Le voyage des Andes des artistes du Rio de la Plata au XXe siècle ", Artelogie, $\mathrm{n}^{\circ} 14,2014$, pp. 1-18.

2. Felipe Cossío del Pomar (1888- 1981) originaire du nord du Pérou fit des études de droit en Belgique et fréquenta Chagall et Picasso avant de partir aux Etats-Unis en 1917 où il se fit portraitiste, Son engagement politique en faveur de l'APRA, l'Alliance Populaire Révolutionnaire Populaire, fondée en 1924 par Víctor Raúl Haya de la Torre, détermina d'une longue vie en exil, au Mexique, où il a fondé l'École des Beaux-Arts de San Miguel de Allende.

3. Rebeca Oquendo, mariée à un diplomate chilien en poste en France, retourne au Pérou en 1879 et se consacre à la peinture et à la formation artistique des jeunes filles en donnant des cours particuliers chez elle. L'œuvre de Rebeca Oquendo est connue par la donation qu'elle fit en 1928 au musée national d'histoire.

4. Elvira Garcia y Garcia évoque longuement le parcours d'Isabel Morales Macedo reçue à l'Académie San Fernando de Madrid après deux années à l'ENBA. Isabel Morales Macedo s'installa définitivement en Espagne.

5. Majluf et Wuffarden Elena Izcue. Lima-Paris Années 30, cité par Cristina Vargas Pacheco «Una visión del Perú a través del arte decorativo», Mercurio peruano, n524, 2011, 151-173.

6. La gravure emblématique d'Amauta est un visage d'Indien paru en couverture du premier numéro (septembre 1926), et réalisé par José Sabogal qui suggéra le nom d'Amauta au lieu d'Avant-garde (Vanguardia). José Carlos Mariátegui, directeur du mensuel, expliqua: «Le titre [Maître/Sage en quechua] traduit notre adhésion à la Race ainsi que notre hommage à l'Incaïsme » (Amauta, « Presentación de Amauta », nº 1, juin 1926, p. 1).

Amauta parut pendant quatre ans (1926-1930, trente numéros) et fut la revue la plus diffusée des débats politiques et culturels d'avant-garde en faveur de l'indigénisme.

7. Le même numéro d'Amauta inclut trois comptes rendus d'expositions, d'une part de Carmen Saco sur les xylographies de Sabogal («Des personnages comme son pêcheur chimu ont selon nous la valeur de la création d'un néo-primitif. Ces xylographies nous présentent avec la même 
vérité la dureté antique des pierres de Cusco et la tendresse fruitée des jeunes indiennes ['cholitas '] ", Amauta, 27, décembre 1929, p. 99), et sur Victor Valdivia défini d'entrée comme « aymara » et célébré pour ses paysages «qui sont la fête du vert juteux, humide et tendre [...] liés par l'ossature de la roche [...] où sont dessinées les silhouettes comme par un coup de pinceau sur du verre » et «Catalogue de l'exposition de Julia Codesido » par Xavier Abril (ibid, p. 100).

8. Il n'y a pas de reproduction de « Cecilia » mis à part dans Amauta, l'original n'est pas localisé.

9. Si Mariátegui s'impose comme l'idéologue du socialisme péruvien, Víctor Raúl Haya de la Torre fédère une partie des intellectuels et des classes populaires dans le projet d'une troisième voie antiimpérialiste et nationaliste, en faveur des groupes opprimés. L'APRA à vocation continentale est créée à Mexico en 1924, aux côtés de Vasconcelos, auteur de La raza cósmica, au nom de l'Amérique métissée.

10. Carlota Carvallo écrit dans son journal intime: «...à la maison, on m'a dit des horreurs après que j'ai montré une photographie de mon portrait. On m'a dit que je suis immorale d'avoir posé ainsi...», (Carlota Carvallo, Diario de 1931, p. 92, inédit). Une copie du journal intime de Carlota Carvallo, épouse de l'intellectuel péruvien Estuardo Nuñez, doit être éditée par la famille.

11. Alicia et Celia Bustamante ouvrirent « la Peña » Pancho Fierro en 1937, comme un lieu de rencontre et d'exposition pour les artistes et les intellectuels, revendiquant la musique et l'art populaire. Cf. Kelly Carpio et María Eugenia Yllia, «Alicia y Celia Bustamante, la Peña Pancho Fierro y el Arte Popular », Illapa, $n^{\circ} 12,201$, pp. 45-60.

12. Eduardo Moll, Julia Codesido, Lima, Navarrete, 1990, p. 33. Un passeport établi en 1935 donnait pour année de naissance 1892, ce qui rajeunissait considérablement Codesido. L'ouvrage de Moll reste à ce jour le plus complet sur Codesido.

13. Les tableaux reproduits dans Amauta sont intitulés en espagnol «Tapicera india ", "Arcilla quechua », « La quena », « Indio aymara », « La huahua », « Retrato del novelista Augusto Aguirre Morales », «Chola cuzqueña » (Amauta, $\mathrm{n}^{\circ}$ 11, janvier 1928, « Arte peruano. Julia Codecido », pp. 9-11). Ève-Marie Fell a fait le décompte des productions visuelles indigénistes dans Amauta: 25 œuvres de Sabogal, 18 de Codesido, 19 de Camilo Blas et 3 de Teresa Carvallo (supra)

14. Jorge Falcón, Julia Codesido, Lima, 1987, Minerva, p. 12.

L'opuscule de Falcón intègre l'entretien de Julia Codesido inclus dans le catalogue de l'exposition de la galerie René Métras de Barcelone (1966).

15. La première promotion de l'École Nationale des Beaux-Arts rassemble une centaine d'élèves dont près de quarante femmes (Moll, 1990, pp. 73-76).

16. G. Buntinx et Luis Eduardo Wuffarden, Mario Urteaga: nuevas miradas, Lima, 2003, Museo de arte de Lima-Telefónica.

Mario Urteaga est un peintre autodidacte qui s'est formé par l'observation et a réalisé son œuvre dans la région nord-andine du Pérou ; Camilo Blas (1903-1985), neveu de Mario Urteaga, suivit la formation de l'ENBA et est considéré comme l'un des disciples de José Sabogal.

17. Les toiles arrivèrent au Petit Palais le 11 mai 1953 ; aucun catalogue n'a été archivé ; les titres en français dans la plaquette et dans le reçu du musée, sont les suivants :

1. Morochuco. 2. Oiseaux bleus. 3. Femmes de l'Amazone. 4. Tondero (danse populaire). 5. Marinera (danse populaire). 6. Cashua (danse populaire). 7. Créole. 8. Paysage des Andes (alpaca). 9. Petite indienne. 10. «Colla» indienne. 11. Colibri. 12. Les condors. 13. Joueurs de quenas. 14. Paysage des Andes. 15. Bergère. 16. Fête. 17. Tête. 18. Masque indien. 19. Indienne et lama. 20. Patronne d'Amancaes. 21. Femmes; 22. Femme à la cruche. 23. Jeune femme au peigne. 24. Procession. 25. Nu. 26. Foire. 27. Cheval vert. 28. Le chemin. 29. Le christ. 30. Les dunes. 31. Chefs indiens. 32. Femmes de la forêt. 33. Le « kero »/le vase.

18. Le tableau est reproduit dans l'ouvrage d'Eduardo Moll $(1990,28)$. 


\section{RÉSUMÉS}

Le présent article situe l'œuvre de la Péruvienne Julia Codesido dans l'indigénisme aux débuts de ce mouvement culturel et social, alors que Codesido comme Sabogal, comme Daniel Hernández reviennent d'Europe au Pérou. Le cheminement inverse du Pérou vers la France est réalisé par Elena Izcue et Carmen Saco dans les années 1920. La revue Amauta dirigée par José Carlos Mariátegui est le fer de lance de l'activité indigéniste à Lima. Plusieurs reproductions de tableaux de Codesido sont publiées correspondant à l'engagement du mensuel. Après avoir présenté ces œuvres, l'article évoque l'évolution de Codesido en reliant l'épuration plastique des années 40-50 à l'activité au sein de l'Institut d'Art Péruvien fondé par José Sabogal.

El presente artículo sitúa la obra de la peruana Julia Codesido en el indigenismo a inicios del movimiento cultural y social cuando Codesido como Sabogal, como Daniel Hernández regresaron de Europa al Perú. Por el contrario, Elena Izcue y Carmen Saco salieron para Francia en los mismos años 1920. La revista Amauta dirigida por José Carlos Mariátegui fue la punta de lanza de la actividad indigenista en Lima. Varias reproducciones de los cuadros de Codesido se publicaron conformes al compromiso de la revista. Después de presentar esas obras, el artículo plantea la evolución de Codesido relacionando la depuración en las artes plásticas de los años 40-50 con la actividad de Codesido en el Instituto de Arte Peruano fundado por José Sabogal.

\section{INDEX}

Mots-clés : Julia Codesido, Pérou, Indigénisme, Carmen Saco, Elena Izcue, José Sabogal

Palabras claves : Julia Codesido, Perú, indigenismo, Carmen Saco, Elena Izcue, José Sabogal

\section{AUTEUR}

\section{ISABELLE TAUZIN-CASTELLANOS}

Professeur d'études latino-américaines de l'université Bordeaux Montaigne, spécialiste du Pérou. Les traductions de l'espagnol dans le présent article sont sous sa responsabilité. Isabelle TauzinCastellanos est membre de l'Institut Universitaire de France. 